\title{
Towards a comparable quantification of resilience
}

\section{Authors}

Ingrisch, Johannes; Bahn, Michael

Address

Institute of Ecology

University of Innsbruck

Sternwartestr. 15

6020 Innsbruck, Austria

Corresponding author

Bahn, M (Michael.bahn@uibk.ac.at)

\section{Keywords}

Disturbance impact, recovery rate, resilience index, resilience metric, resistance, recovery time

\section{Highlights}

- A common framework for a comparable quantification of resilience is missing

- Previous resilience metrics do not permit standardized comparisons across systems

- Metrics need to adequately account for the role of resistance and recovery

- A bivariate framework considering the components of resilience has great potential. 


\section{Abstract}

Resilience is a key concept in ecology and describes the capacity of an ecosystem to maintain its state and recover from disturbances. Numerous metrics have been applied to quantify resilience over a range of ecosystems. However, the way resilience is quantified affects the degree to which different trajectories of ecosystem recovery from disturbance are represented as 'resilient', precluding a comparison of disturbance responses across ecosystems and their properties and functions. To approach a broadly comparable assessment of resilience we suggest using a bivariate framework, which jointly considers the disturbance impact and the recovery rate, both normalized to the undisturbed state of a system. We demonstrate the potential of the framework for attributing and integrating across the various components underlying resilience.

\section{The need for a common currency of resilience}

For decades, ecologists have used the concept of 'resilience' (see Glossary) to describe the manifold responses of ecosystems to disturbances (e.g. climate extremes) [1]. Disturbances can cause transient changes in ecosystem properties and processes (e.g. temporary changes of productivity [2-4]) or even lead to a complete reorganization of an ecosystem by causing a shift to an alternative stable state [5], e.g. from forest to savannah [6]. Accordingly, two complementary perspectives on resilience have emerged. The first focuses on the transient impact of disturbance and the subsequent recovery of an ecosystem and has been termed 'engineering resilience' (e.g. [7-9]). The second view considers resilience as the ability of an ecosystem to withstand a shift to an alternative state in the face of disturbance ('ecological resilience') $[9,10]$. The increasing recognition of the implications of disturbances for ecosystems and society has recently stimulated an extensive debate on the concept of resilience and its components $[1,8,11-15]$, with partly contradictory definitions and terminologies proposed along $[8,12]$ and beyond [16-20] the classical views described above. While some of this debate has focused on semantics, it has also brought forth a much needed exploration of our understanding of the mechanisms underlying resilience $[8,11,15,16,21,22]$.

The attempt to quantify ecosystem responses to disturbance has led to the development of a large number of 'indicators' or 'metrics' of resilience, which are the most stringent formal expression of how researchers define and quantify resilience and its components. A recent review of indicators of ecological resilience summarized possible approaches to detecting critical transitions between alternative stable states and highlighted that only a few of the theoretically suggested metrics have been explored in field situations [23]. In contrast, a broad range of metrics have been applied to 
observational and experimental studies for quantifying ecosystem recovery from disturbance within a given stability domain (i.e. 'engineering resilience'). However, it is unclear to what degree these different metrics are actually comparable and whether any of them could serve as a common currency for a comparable quantification of the resilience of ecosystems. It would be essential to identify a broadly comparable approach assessing which ecosystems, and which ecosystem properties and processes, are more resilient than others, across various types, intensities and frequencies of disturbances. In view of the pressing need to advance the understanding of resilience beyond isolated case studies and to provide broader conclusions for science and society, a common language and a common currency for resilience are urgently needed.

\section{Quantifying resilience - how do the metrics compare?}

The metrics of resilience of ecosystem response to disturbance within a stability domain quantify the capacities to resist and to recover from disturbance (Box 1) (note that in this paper we adhere to the resilience terminology used by Hodgson et al. [12]). The metrics applied so far in the literature can be grouped to three major types, which differ fundamentally depending on the component of resilience they address (Table 1). The simplest category (Category I) comprises metrics describing the impact of a disturbance on an ecosystem state (or process rate) in relation to the baseline (Box 1, Figure IB). The baseline is typically considered the pre-disturbance state, but in factorial experiments can also be derived from simultaneous observations in an undisturbed control treatment. More complex metrics focus primarily on recovery, i.e. on the amount by which the system has recovered (post-disturbance change in state). Recovery from disturbance can be expressed relative to the baseline (Category II) or relative to the disturbance impact (Category III). Thereby, the metrics falling into Category II indicate by which percentage of the pre-disturbance state the system has recovered, whereas indices of Category III indicate how much of the disturbance impact has been recovered (see Box 1).

Unfortunately, various studies have applied the same terminology across all categories (Table 1). Furthermore, across categories and even within each category, the different equations used in different studies lead to very different quantifications of any given response trajectory (Figure 1). For example, in Category I, full recovery from disturbance implies convergence of the metric to 0 or to 1 , depending on how authors calculate resilience (Table 1, Figure 1A, B). Similarly, in Category III full recovery has also been denoted by either 0 or 1 (Table 1), and can even tend towards infinity (Figure 1F). This is not only problematic in terms of a comparison of metrics across studies. An equation inflating a metric towards infinity as the system recovers can also potentially overemphasize effects of e.g. biodiversity on ecosystem resilience to drought [24]. Different approaches to calculating a metric of resilience can lead to diverging conclusions when comparing response trajectories representing different ecosystems, different types of disturbances or different ecosystem state variables. For 
example, at a given point in time $\left(\mathrm{t}_{\mathrm{x}}\right)$, systems $\boldsymbol{a}$ and $\boldsymbol{c}$ differ in the metrics of Category I and III, but are identical according to Category II (Figure 1); system $\boldsymbol{d}$ can be more or less resilient than system $\boldsymbol{c}$, depending on the metric applied (Figure 1). This results from the fact that trajectory $\boldsymbol{d}$ has a higher rate of baseline-normalized recovery, but a lower rate of impact-normalized recovery than trajectory $c($ Box 1).

Our survey of previously used metrics therefore indicates that the choice of the metric, i.e. the way resilience is quantified, affects the degree to which different trajectories of ecosystem recovery from disturbance are represented as 'resilient'. In consequence, the fundamental differences between metrics concerning the way they have been calculated preclude any comparative analysis of ecosystem resilience to disturbances across the studies published so far. We furthermore argue that previously applied single indices do not adequately account for the fact that resistance to and recovery from disturbance can jointly and independently influence resilience $[8,12]$. This holds similarly for recently adopted area-based metrics of resilience $[13,25,26]$, which primarily quantify aspects of perturbation (see Glossary and Box 1). Also recovery time (e.g. [27]), albeit intuitive and informative, is not recommendable as a single metric for resilience, as it does not attribute the relative importance of resistance and recovery and may be associated with variable levels of perturbation (see Fig. 2B and related text).

To date, only few studies have jointly considered multiple attributes of recovery $[13,28-30]$. While the application of multiple metrics can be powerful for understanding resilience in multiple contexts, it precludes a standardized comparison of different ecosystems and the consideration of most previous studies on the topic [31]. To make progress towards a generally comparable assessment of engineering resilience across different ecosystems it would be essential to develop approaches, which account for resistance and recovery in a coherent framework.

\section{A bivariate framework for comparing resilience}

We propose a quantitative assessment and comparison of resilience, which integrates the major components underlying resilience into a single framework (Fig. 2B). This framework adopts the recent suggestions by Hodgson et al. [12] and Nimmo et al. [8] to map resilience bivariately based on a joint consideration of the resistance and the recovery of a system. While Hodgson et al. plot return time in relation to change in state, Nimmo et al. relate the percent change from before to the end of a disturbance (resistance) to the percent change in state during recovery. Here, we propose to use the normalized impact of disturbance and the normalized recovery rate for defining the bivariate space. We argue that a normalization of impact and recovery rate is essential for obtaining a coherent comparison of resilience across different systems. We suggest that normalization is made in relation 
to the baseline state of a system (Box 1). Plotting the baseline-normalized recovery rate of a system in relation to the baseline-normalized impact permits deriving the recovery time (Fig. 2B), and, conversely, the impact-normalized recovery rate of a system (see Box 1 and Supplement). The proposed scheme also considers perturbation, which integrates the total reduction in ecosystem state or function over the recovery time (Box 1). Importantly, each point in the bivariate space corresponds to a unique combination of impact and recovery rate, and, in consequence, of recovery time and perturbation (Fig. 2B). Thus, the proposed framework relates all key parameters underlying resilience in a quantitative way and thereby permits a coherent comparison of disturbance responses across ecosystems.

To illustrate how the suggested framework permits a comparison of resilience and its components we depict twelve cases (Figure $2 \mathrm{~A}$ ) representing all possible combinations of response trajectories of ecosystems to disturbance relative to the given disturbance response of a reference system (dashed line in panels of Figure 2A). In our examples, the state of the reference system (e.g. biomass) was reduced by $50 \%$ upon disturbance and recovered to the baseline state within 25 days. In comparison, a system suffering only $25 \%$ reduction of its state and recovering after 10 days is characterized by a higher resistance (and thus lower disturbance impact) and a higher recovery rate compared to the reference system (case 1). The reverse holds for case 12.

In most cases the resilience of a system scales with the recovery time, i.e. systems returning faster to their baseline state are also more resilient. However, while cases 4 and 9 exhibit the same recovery time as the reference system there is an arguable difference in their resilience: in case 4 both the disturbance impact and the recovery rate are lower compared to the reference system, thus the overall perturbation caused by the disturbance is smaller (Fig. 2B) and the system considered in case 4 is more resilient than the reference system. The reverse holds for case 9. These two examples demonstrate that recovery time, or perturbation, alone are not sufficient for an unambiguous comparison of the resilience of different systems (see also previous section).

When mapped onto the bivariate response space the four disturbance responses $\boldsymbol{a}-\boldsymbol{d}$, which were already used as examples in Box 1 and Fig. 1, can be directly compared concerning their resilience and the underlying processes (Fig. 2C): system $\boldsymbol{d}$ is more resilient than system $\boldsymbol{a}$ due to a higher resistance (lower normalized impact) and a higher baseline-normalized recovery rate, and thus a shorter recovery time (see case 1). System $\boldsymbol{b}$ is less resilient than system $\boldsymbol{c}$ in spite of an identical recovery time, because of a higher perturbation (see case 9).

In a similar fashion the proposed framework can be applied to real-world examples corresponding to the cases shown in Fig. 2B. Studying the resilience of soil microbial activity following a dry-wet cycle 
Orwin and Wardle [32] observed a lower resistance and a higher baseline-normalized recovery rate for soils with low as compared to high organic matter content. This situation is represented by case 8 (i.e. lower resilience). Analyzing the drought responses of ecosystem photosynthesis of mountain grassland, Ingrisch et al. [33] found that abandoned grassland was more resistant and had a lower baseline-normalized recovery rates than managed grassland. While the recovery time was identical in both grasslands, the perturbation was lower in the abandoned grassland, which was therefore more resilient (case 4). Another recent study showed that elevated $\mathrm{CO}_{2}$ did not alter the impact of an experimental drought combined with a heatwave on grassland carbon uptake, but increased its recovery rate ([34], case 6). Testing for effects of species richness on the resilience of biomass to drought, van Ruijven and Berendse [29] and Kreyling et al. [35] observed no effect of species richness on the normalized drought impact, but an increase of recovery rate with increasing species richness (case 6). It should be noted that these latter two biomass-based studies measured recovery only in the year following the extreme event, so strictly speaking recovery rates cannot be derived and therefore resilience can at best be approximated.

It is important to point out that the position of a response within the bivariate framework can potentially change in the case of nonlinear recovery responses, i.e. when recovery rates change over time. The detection of nonlinear responses requires several measurements during the recovery period, which have so far been rarely available, especially for the many studies targeting biomass as a state variable. In the bivariate scheme (Fig. 2) recovery responses with a low degree of nonlinearity could be represented by a single position reflecting an average recovery rate. However, in the case of pronounced nonlinearities it may be more instructive to plot the temporal changes in recovery rates by sequential positions in the bivariate space. Thus, for example, a downward shift in sequential positions (e.g. from 1 to 5 or from 8 to 12 in Fig. 2B) would indicate a progressive slowing down of the recovery rate.

While it is targeted towards a consistent comparison of the engineering resilience of ecosystems, the bivariate scheme also provides a broad indication of the risk of an ecosystem crossing a tipping point towards an alternative state. The risk increases when exceptionally high recovery times and perturbations occur (lower right end of the scheme in Fig. 2B), both associated with low recovery rates. However, the bivariate scheme cannot specify the precise location of any tipping point. This is due to the fact that the perturbation leading to a tipping point cannot be standardized across systems, and ultimately depends on the mortality thresholds and population dynamics of species and other intrinsic factors of an ecosystem, as well as the legacy, and the frequency and severity of previous disturbance events [36-38]. To be able to predict tipping points comprehensive datasets with long time-series are required that permit the quantification of a critical slowing down of a system $[23,39]$. While recovery 
rates have been suggested to be a proxy for ecological resilience in some cases [23], the challenge remains to identify metrics reconciling the concepts of ecological and engineering resilience and make them applicable to (e.g. experimental) studies yielding comparatively small and short-term datasets.

We conclude that a quantitative assessment of resilience can contribute to resolving apparent and real contradictions in the conceptualization of resilience. The suggested bivariate framework could serve for a consistent comparison of resilience across ecosystems and disturbances, while at the same time attributing the respective roles of resistance and recovery. The framework can also be used for comparing the resilience of different ecosystem properties and processes. This can be useful when evaluating how disturbances affect the multiple functional responses of ecosystems and which management practices are best suited for enhancing the resilience of specific ecosystem services (such as carbon sequestration, timber production or water quality) while minimizing the risk of disturbance impacts on others [40]. The proposed framework could thus contribute to a more coherent comparison of the responses and vulnerabilities of ecosystems to disturbances in a rapidly changing world. 


\section{Box 1: Quantifying the components of resilience}

A resilient ecosystem is able to minimize the impact of a disturbance and to recover from the impact. The disturbance impact can be quantified by the change in ecosystem state, i.e. the difference between the pre-disturbance state and the state at the time of peak impact (Fig. IA). The recovery rate can be quantified by the change of ecosystem state per unit time after disturbance. Both the impact and the recovery rate determine the recovery time (Fig. IA): the smaller the impact and the higher the recovery rate, the smaller the perturbation will be (gray area in Fig. IA) and the more quickly an ecosystem will recover.

When comparing different ecosystems it is meaningful to compare the disturbance responses relative to the pre-disturbance state of respective system. This can be achieved by expressing the system state after disturbance in percent of its state before disturbance (baseline state; Fig. IB). Baseline normalization accounts for background differences between ecosystems prior to disturbance. For example, in response to disturbance system $\boldsymbol{b}$ suffers a larger impact than system $\boldsymbol{a}$ (Fig. ID). However, as the pre-disturbance state of system $\boldsymbol{b}$ is also larger, the baseline-normalized impact on the two systems is the same (Fig IE). Similarly, while the recovery rate for system $\boldsymbol{c}$ is higher than for system $\boldsymbol{a}$ in absolute terms (Fig. ID), it is identical when considering its higher baseline state (Fig. IE). Baselinenormalization of ecosystem states following disturbance has been frequently used for deriving metrics of resilience (see Table 1).

To be able to compare the recovery of systems independent of disturbance impact, some studies have normalized the recovery of the system state to impact (Fig. IC, Table 1), defining the system state at peak impact as $0 \%$ and the baseline state as $100 \%$. For mathematical reasons (see Supplementary Material) the impact-normalized recovery rate is the reciprocal of the recovery time. Thus, in our example, the two systems with the highest, and identical, impact-normalized recovery rates (systems $\boldsymbol{b}$ and $\boldsymbol{c}$ ) (Fig. IF) exhibit the lowest, and identical, recovery times (Fig. ID). 


\section{Box 2: Outstanding questions}

- How can the resilience of different ecosystems be compared and the role of its component processes be interpreted in a standardized way? How does the type and severity of a disturbance affect resilience? Which ecosystems are most strongly impacted by which kind of disturbances and which are most resilient?

- How can the resilience of different properties and functions of a given ecosystem be compared? Which properties and functions are most vulnerable, and which are most resilient? How does the resilience of different ecosystem properties and functions relate? Which resilience trade-offs exist between different ecosystem properties and functions?

- How can the concepts and metrics of engineering resilience and ecological resilience be merged into a coherent framework? 


\section{Glossary}

Baseline state: state of an ecosystem before disturbance.

Impact: change of ecosystem state or functions caused by a disturbance. Impact can be expressed in absolute terms or relative to the pre-disturbance state (baseline-normalized).

Perturbation: cumulative reduction in ecosystem state or function integrated over the recovery time (see Box 1, gray area in Fig IA).

Recovery: capacity of an ecosystem to return to undisturbed ecosystem state and functioning following a disturbance.

Recovery rate: rate at which an ecosystem state or function recovers from disturbance impact. It can be expressed in absolute terms, relative to the pre-disturbance state (baseline-normalized) or relative to the disturbance impact (impact-normalized).

Resilience: capacity of an ecosystem to persist and maintain its state and functions in the face of disturbance. It is determined by the capacity to reduce the impact (resistance) and the capacity to recover from the impact of disturbance (recovery).

Resistance: ability to persist during the disturbance, measureable through the concurrent impact of a disturbance on response parameters. The higher the resistance the smaller is the impact.

Response trajectory: temporal change of ecosystem state or function during and after a disturbance.

Recovery time: time from the end of disturbance to the full recovery of an ecosystem state or function.

\section{Acknowledgements}

This work emerged from the ERA-Net BiodivERsA project 'REGARDS' (Austrian Science Fund project no I 1056) and was co-funded by Austrian Science Fund project no. P28572 and the Austrian Academy of Sciences (project ClimLUC). J.I. was supported by a Ph.D. completion grant from the University of Innsbruck. 


\section{References}

1 Standish, R.J. et al. (2014) Resilience in ecology. Abstraction, distraction, or where the action is? Biol. Conserv. 177, 43-51

2 Hoover, D.L. et al. (2014) Resistance and resilience of a grassland ecosystem to climate extremes. Ecology 95, 2646-2656

3 Ciais, P. et al. (2005) Europe-wide reduction in primary productivity caused by the heat and drought in 2003. Nature 437, 529-533

4 Wright, A.J. et al. (2015) Flooding disturbances increase resource availability and productivity but reduce stability in diverse plant communities. Nat. Commun. 6, 6092

5 Scheffer, M. et al. (2001) Catastrophic shifts in ecosystems. Nature 413, 591-596

6 Hirota, M. et al. (2011) Global resilience of tropical forest and savanna to critical transitions. Science 334, 232-235

7 Pimm, S.L. (1984) The complexity and stability of ecosystems. Nature 307, 321-326

8 Nimmo, D.G. et al. (2015) Vive la resistance: reviving resistance for 21st century conservation. Trends Ecol. Evol. 30, 516-523

9 Holling, C.S. (1996) Engineering resilience versus ecological resilience. In Engineering within ecological constraints (Schulze, P., ed), pp. 31-44, National Academy Press

10 Gunderson, L.H. (2000) Ecological Resilience-In Theory and Application. Annu. Rev. Ecol. Syst. 31, 425-439

11 Oliver, T.H. et al. (2015) Biodiversity and Resilience of Ecosystem Functions. Trends Ecol. Evol. 30, 673-684

12 Hodgson, D. et al. (2015) What do you mean, 'resilient'? Trends Ecol. Evol. 30, 503-506

13 Todman, L.C. et al. (2016) Defining and quantifying the resilience of responses to disturbance: a conceptual and modelling approach from soil science. Scientific reports 6, 28426

14 Quinlan, A.E. et al. (2016) Measuring and assessing resilience. Broadening understanding through multiple disciplinary perspectives. J. Appl. Ecol. 53, 677-687

15 Angeler, D.G. and Allen, C.R. (2016) Quantifying resilience. J. Appl. Ecol. 53, 617-624

16 Yeung, A.C.Y. and Richardson, J.S. (2016) Some Conceptual and Operational Considerations when Measuring 'Resilience': A Response to Hodgson et al. Trends Ecol. Evol. 31, 2-3

17 Mori, A.S. (2016) Resilience in the Studies of Biodiversity-Ecosystem Functioning. Trends Ecol. Evol. $31,87-89$

18 Oliver, T.H. et al. (2016) A Synthesis is Emerging between Biodiversity-Ecosystem Function and Ecological Resilience Research: Reply to Mori. Trends Ecol. Evol. 31, 89-92

19 Sundstrom, S.M. et al. (2016) Resisting Resilience Theory: A Response to Connell and Ghedini. Trends Ecol. Evol. 31, 412-413

20 Connell, S.D. et al. (2016) Ecological Resistance - Why Mechanisms Matter: A Reply to Sundstrom et al. Trends Ecol. Evol. 31, 413-414

21 Connell, S.D. and Ghedini, G. (2015) Resisting regime-shifts: the stabilising effect of compensatory processes. Trends Ecol. Evol. 30, 513-515

22 Song, H.-S. et al. (2015) Integrating Ecological and Engineering Concepts of Resilience in Microbial Communities. Front. Microbiol. 6, 1298

23 Scheffer, M. et al. (2015) Generic Indicators of Ecological Resilience. Inferring the Chance of a Critical Transition. Annu. Rev. Ecol. Evol. Syst. 46, 145-167

24 Isbell, F. et al. (2015) Biodiversity increases the resistance of ecosystem productivity to climate extremes. Nature 526, 574-577 
25 Hagedorn, F. et al. (2016) Recovery of trees from drought depends on belowground sink control. Nature plants 2, 16111

26 Zhang, B. et al. (2010) Does microbial habitat or community structure drive the functional stability of microbes to stresses following re-vegetation of a severely degraded soil? Soil Biol. Biochem. 42, 850-859

27 Schwalm, C.R. et al. (2017) Global patterns of drought recovery. Nature 548, 202-205

28 Lloret, F. et al. (2011) Components of tree resilience. Effects of successive low-growth episodes in old ponderosa pine forests. Oikos 120, 1909-1920

29 van Ruijven, J. and Berendse, F. (2010) Diversity enhances community recovery, but not resistance, after drought. J. Ecol. 98, 81-86

30 Banning, N.C. and Murphy, D.V. (2008) Effect of heat-induced disturbance on microbial biomass and activity in forest soil and the relationship between disturbance effects and microbial community structure. Appl. Soil Ecol. 40, 109-119

31 Hodgson, D. et al. (2016) Resilience Is Complicated, but Comparable. A Reply to Yeung and Richardson. Trends Ecol. Evol. 31, 3-4

32 Orwin, K.H. and Wardle, D.A. (2004) New indices for quantifying the resistance and resilience of soil biota to exogenous disturbances. Soil Biol. Biochem. 36, 1907-1912

33 Ingrisch, J. et al. (2017) Land Use Alters the Drought Responses of Productivity and CO2 Fluxes in Mountain Grassland. Ecosystems 77, 118

34 Roy, J. et al. (2016) Elevated $\mathrm{CO} 2$ maintains grassland net carbon uptake under a future heat and drought extreme. Proceedings of the National Academy of Sciences of the United States of America $113,6224-6229$

35 Kreyling, J. et al. (2017) Species richness effects on grassland recovery from drought depend on community productivity in a multisite experiment. Ecology letters

36 Bahn, M. et al. (2014) Climate-biosphere interactions in a more extreme world. The New phytologist 202, 356-359

37 Smith, M.D. (2011) An ecological perspective on extreme climatic events. A synthetic definition and framework to guide future research. J. Ecol. 99, 656-663

38 Luo, Y. et al. (2015) Predictability of the terrestrial carbon cycle. Glob. Chang. Biol. 21, 1737-1751

39 Dakos, V. et al. (2014) Resilience indicators. Prospects and limitations for early warnings of regime shifts. Phil. Trans. R. Soc. B 370, 20130263

40 Seidl, R. et al. (2016) Searching for resilience: addressing the impacts of changing disturbance regimes on forest ecosystem services. J. Appl. Ecol. 53, 120-129

41 Kaufman, L.H. (1982) Stream aufwuchs accumulation. Disturbance frequency and stress resistance and resilience. Oecologia 52, 57-63

42 MacGillivray, C.W. and Grime, J.P. (1995) Testing Predictions of the Resistance and Resilience of Vegetation Subjected to Extreme Events. Funct. Ecol. 9, 640

43 Griffiths, B.S. et al. (2000) Ecosystem response of pasture soil communities to fumigation-induced microbial diversity reductions. An examination of the biodiversity-ecosystem function relationship. Oikos 90, 279-294

44 Griffiths, B.S. and Philippot, L. (2013) Insights into the resistance and resilience of the soil microbial community. FEMS Microbiol. Rev. 37, 112-129

45 Sousa, W.P. (1980) The responses of a community to disturbance. The importance of successional age and species' life histories. Oecologia 45, 72-81

46 Tilman, D. (1996) Biodiversity. Population Versus Ecosystem Stability. Ecology 77, 350-363 


\section{Table}

Table 1: Overview of currently available indices for engineering resilience, grouped into different categories, according to the component of recovery they address.

\begin{tabular}{|c|c|c|c|c|c|c|}
\hline Nr. & Category & Attribute of recovery & Source & Equation $^{a}$ & $\begin{array}{l}\text { Referred to } \\
\text { as }\end{array}$ & Note \\
\hline$\# 1$ & 1 & $\begin{array}{l}\text { Normalized system } \\
\text { state }\end{array}$ & {$[28,29,41]$} & $\frac{P_{x}}{C_{0}}$ & resilience & converges to 1 for full recovery \\
\hline$\# 2$ & 1 & $\begin{array}{l}\text { Normalized system } \\
\text { state }\end{array}$ & [42] & $\frac{P_{x}}{C_{x}}$ & resilience & Converges to 1 for full recovery \\
\hline$\# 3$ & 1 & $\begin{array}{l}\text { Normalized system } \\
\text { state }\end{array}$ & [43] & $\left(\frac{C_{x}-P_{x}}{C_{x}}\right) \times 100 \%$ & resilience & $\begin{array}{l}\text { equation cited incorrectly in } \\
{[32,44] \text {, converges to } 0 \text { for full }} \\
\text { recovery }\end{array}$ \\
\hline$\# 4$ & 1 & $\begin{array}{l}\text { Normalized system } \\
\text { state }\end{array}$ & [30] & $-100\left[\frac{C_{x}-P_{x}}{C_{x}}\right]$ & resilience & Resilience at $t x$ \\
\hline$\# 5$ & II & $\begin{array}{l}\text { Baseline-norm. } \\
\text { recovery }\end{array}$ & [8] & $\frac{P_{x}}{C_{0}}-\frac{P_{i}}{C_{i}}$ & resilience & $\begin{array}{l}\text { Index-value for full recovery } \\
\text { depends on resistance }\end{array}$ \\
\hline \#6 & ॥ & $\begin{array}{l}\text { Baseline-norm. } \\
\text { recovery }\end{array}$ & [28] & $\frac{P_{x}-P_{i}}{C_{0}}$ & $\begin{array}{l}\text { relative } \\
\text { resilience }\end{array}$ & \\
\hline$\# 7$ & $\|$ & $\begin{array}{l}\text { Baseline-norm. } \\
\text { recovery }\end{array}$ & [30] & $\begin{array}{l}-\left[-100\left(\frac{C_{i}-P_{i}}{C_{i}}\right)\right. \\
\left.+100\left(\frac{C_{x}-P_{x}}{C_{x}}\right)\right]\end{array}$ & $\begin{array}{l}\text { relative } \\
\text { resilience }\end{array}$ & \\
\hline$\# 8$ & III & $\begin{array}{l}\text { Impact-norm. } \\
\text { recovery }\end{array}$ & {$[45]$} & $\frac{C_{x}-P_{x}}{C_{i}-P_{i}}$ & $\begin{array}{l}\text { Power of } \\
\text { recovery }\end{array}$ & Limit is 0 for full recovery \\
\hline$\# 9$ & III & $\begin{array}{l}\text { Impact-norm. } \\
\text { recovery }\end{array}$ & [46] & $\frac{C_{0}-P_{x}}{C_{0}-P_{i}}$ & resilience & \\
\hline$\# 10$ & III & $\begin{array}{l}\text { Impact-norm. } \\
\text { recovery }\end{array}$ & [24] & $\left|\frac{P_{i}-C_{0}}{P_{x}-C_{0}}\right|$ & resilience & $\begin{array}{l}\text { Full recovery tends to infinity, } \\
\text { increasing with higher } \\
\text { recovery, symmetric }\end{array}$ \\
\hline \#11 & III & $\begin{array}{l}\text { Impact-norm. } \\
\text { recovery }\end{array}$ & [32] & $\frac{2\left|C_{i}-P_{i}\right|}{\left(\left|C_{i}-P_{i}\right|+\left|C_{x}-P_{x}\right|\right)}-1$ & resilience & $\begin{array}{l}\text { Restricted between }-1 \text { and } 1 \text {, } \\
\text { full recovery=1, increasing with } \\
\text { higher recovery; symmetric }\end{array}$ \\
\hline
\end{tabular}

${ }^{\mathrm{a}} \mathrm{C}$ and $\mathrm{P}$ are the ecosystem state or process rate of the undisturbed control system and a disturbed system, respectively, at a given point in time since disturbance $(\mathrm{t}=\mathrm{x})$, whereby $\mathrm{t}=0$ and $\mathrm{t}=\mathrm{i}$ correspond to time immediately before and immediately after disturbance, respectively. 


\section{Figures}
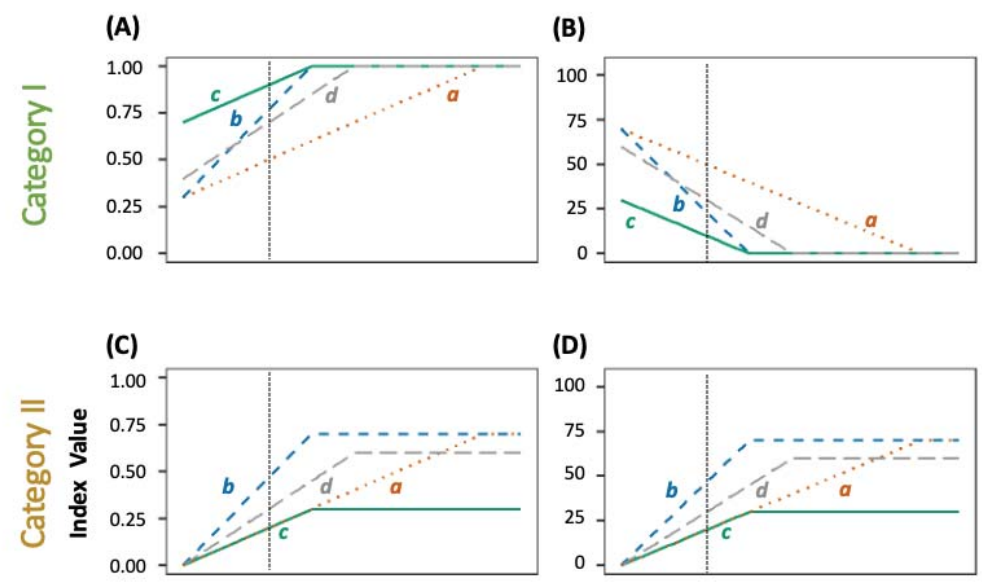

(E)

(F)
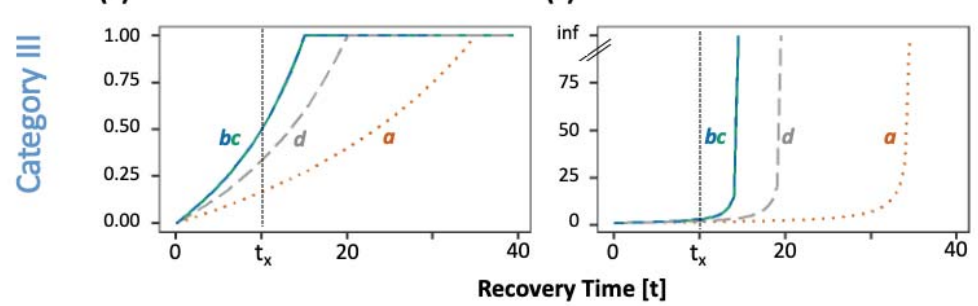

Figure 1. Temporal dynamics of the index values for different recovery metrics, comparing four different hypothetical response trajectories. The systems ( $\boldsymbol{a}-\boldsymbol{d}$, also see Fig I Box 1 ) differ in initial system state resistance and recovery rates, whereby system $\boldsymbol{a}$ and $\boldsymbol{b}$ are characterized by the same resistance, $\boldsymbol{a}$ and $\boldsymbol{c}$ have the same recovery rate relative to baseline, and $\boldsymbol{b}$ and $\boldsymbol{c}$ exhibit the same recovery rate in relation to the disturbance impact (see Box 1). Metrics are grouped according to the categories listed in Table 1, and are based on the equations (A) \# 1 by Kaufman et al. [41] , B) \#2 Griffiths et al. [43], C) \#5 Nimmo et al. [8], D) \#7 Banning and Murphy [30], (E) \#11 Orwin and Wardle [32], (F) \#10 Isbell et al. [24]. Arrows along the x-axis indicate the return time of the corresponding systems, the dotted vertical line at $t_{x}$ indicates a time during the recovery phase at which the systems are compared. 

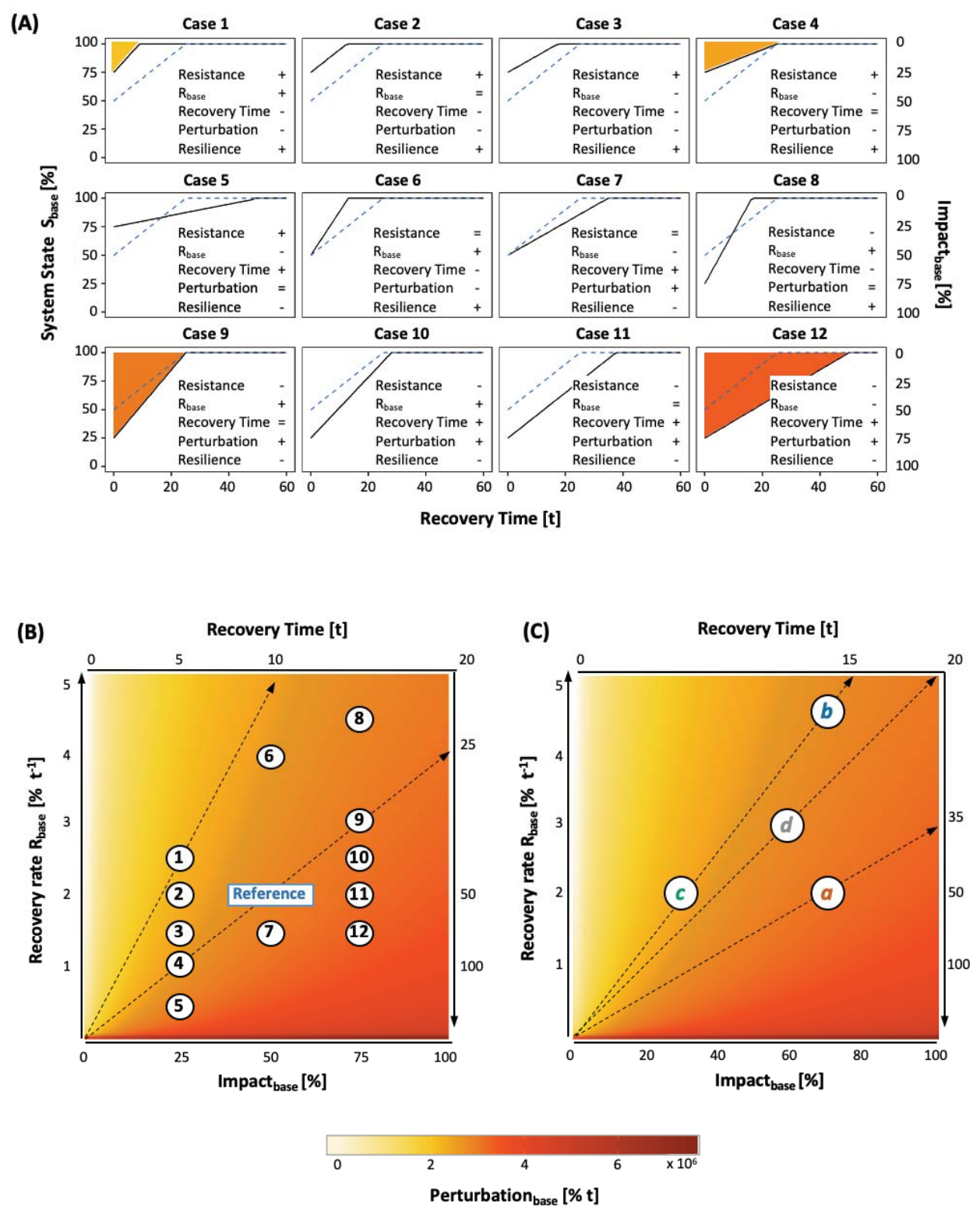

Figure 2: Bivariate framework for a comparable quantification of resilience. (A) Comparing the resilience and its components of a target system (solid line) against a reference system (dashed line). System state and impact are normalized to pre-disturbance state (baseline). The 12 cases represent all possible combinations of systems differing in their resistance and their recovery rates. Insets in each panel indicate whether resilience and its components are higher (+) or lower (-) for the target relative to the reference response. Components include resistance, baseline-normalized recovery rate $\left(R_{\text {base }}\right)$, recovery time and perturbation. Perturbation is graphically illustrated for cases 1, 4, 9 and 12 and relates to the colour scheme in Fig. 2B. For definitions see Glossary and Box 1.

(B) Bivariate scheme for comparing resilience based on disturbance impact and recovery rate, both normalized to the pre-disturbance state of a system (baseline). 1-12 depict the resilience of the 12 cases shown in Fig. 2A. Any combination of baseline-normalized impact and -recovery rate results in a unique combination of recovery time (dashed arrow) and perturbation (background colour).

(C) Applying the bivariate scheme for a resilience assessment of the response trajectories $\boldsymbol{a}$ - $\boldsymbol{d}$ presented in Box 1. 
(A) Absolute

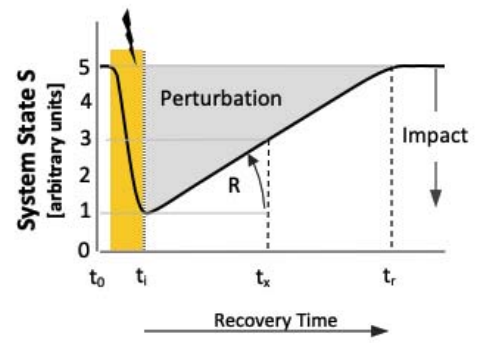

(D)

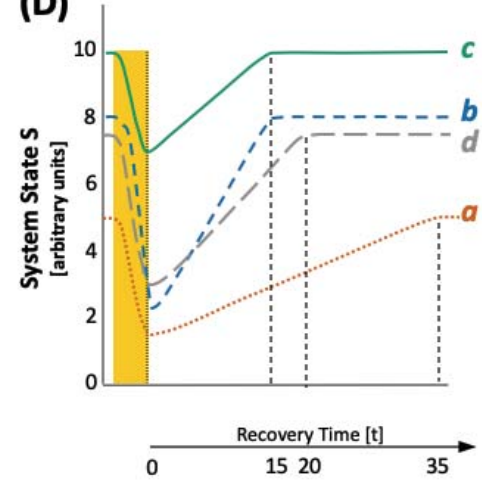

(B) Baseline-normalized

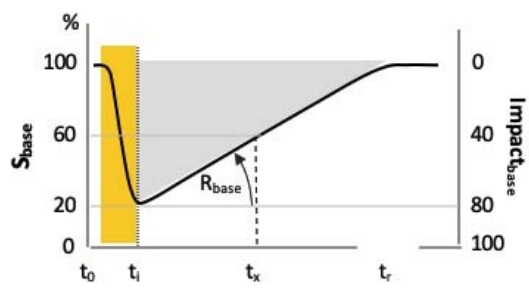

(E)

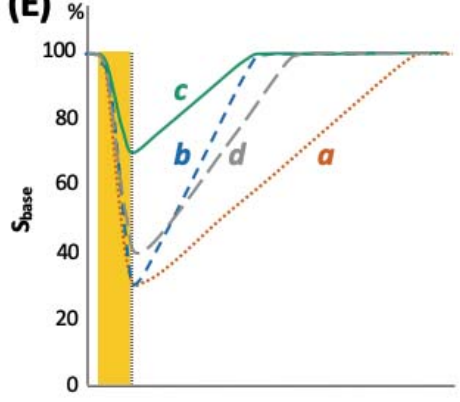

(C) Impact-normalized

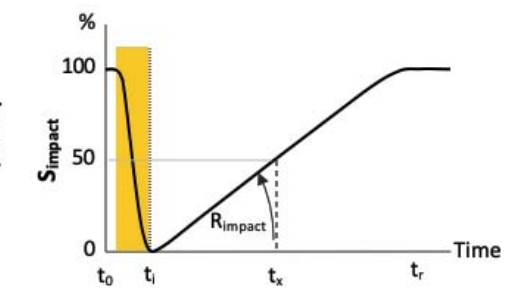

(F)

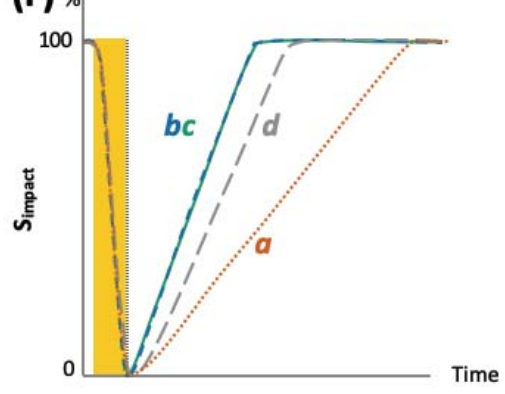

Figure I: Disturbance responses and the main components of resilience

(A) Absolute change in system state (S) over time following a disturbance. Impact and recovery rate $(R)$ jointly determine the time $(t)$ it takes for the system to recover its pre-disturbance state (recovery time). The perturbation (grey area) caused by the disturbance corresponds to the total reduction in ecosystem state integrated over the recovery time.

(B) Disturbance response of a system relative to the pre-disturbance state (=baseline, at time $t_{0}$ ) of the system (baseline-normalization). Both the impact and the recovery rate are expressed in $\%$ of the baseline state.

(C) System recovery from disturbance in \% of the disturbance impact (impact-normalization).

(D) - (F) Examples for four hypothetical response trajectories $\boldsymbol{a}$ - $\boldsymbol{d}$ differing in their baselines, impacts and recovery rates, expressed as absolute changes in state (D), as baseline-normalized changes in state $(E)$ and as impact-normalized changes in state during recovery (F). 